Article

\title{
Isolation and Characterization of the PISTILLATA Ortholog Gene from Cymbidium faberi Rolfe
}

\author{
Yue Fei and Zhi-Xiong Liu * \\ College of Horticulture and Gardening, Yangtze University, Jingzhou 434025, China \\ * Correspondence: zxliu@yangtzeu.edu.cn; Tel.: +86-716-8066260
}

Received: 29 May 2019; Accepted: 1 August 2019; Published: 2 August 2019

check for updates

\begin{abstract}
Cymbidium faberi Rolfe is a very popular potted plant in China, Japan and Korea where it has been cultivated for centuries. The economic value of this popular native Asian orchid could be enhanced by changes in its floral traits. In Arabidopsis, PISTILLATA (PI) is involved in regulating petal and stamen development. In order to investigate the possible role of the PI ortholog involved in floral development, we isolated CyfaPI from C. faberi. Protein alignment and a phylogenetic tree grouped CyfaPI in the PI lineage. CyfaPI transcripts were detected in all floral organs, but were absent in leaves. Moreover, in flowers, the highest expression level of CyfaPI was present in the gynostemium and the lowest level was found in anther caps. In addition, ectopic expression of CyfaPI in Arabidopsis pi-1 mutant rescued petal development, and complement the development of filament-like structure (part of stamen), but failed to complement anther development in the stamen whorl. All these finding suggest that CyfaPI is mainly responsible for perianth and gynostemium development in C. faberi. Our data may help to trace the development of the gynostemium program and evolution in orchids.
\end{abstract}

Keywords: PISTILLATA; Cymbidium faberi; MADS-box gene; Floral development; Orchidaceae

\section{Introduction}

Cymbidium faberi Rolfe is a very popular potted plant in several countries of East Asia, and has been cultivated for centuries in China, Japan and Korea. Many artificially propagated varieties have been developed based on various flower traits including their color and number as well as the form of the perianth (sepal and petal). All of these elements contribute to the visual appreciation of orchids. The perianth is the most visually obvious structure, which is related to its primarily function as an optical attraction for pollinators. Moreover, the molecular mechanisms underlying the perianth identity program have been intensively studied in model species such as Arabidopsis thaliana, Antirrhinum majus and Petunia hybrida [1], and the evolution of the perianth identity has been extensively discussed recently in [2]. However, the perianth in monocots does not completely correspond to the obvious differentiation of sepal and petal whorls in model eudicots. In eudicots, the conserved function of the PISTILLATA (PI)/GLOBOSA (GLO) orthologs, such as MtPI from Medicago truncatula [3], TrPI from Taihangia rupestris [4], CabuPI from Catalpa bungei [5], is the regulation of petal and stamen development. Furthermore, each ortholog is able to fully complement the floral phenotype of the Arabidopsis pi mutant, and the expression pattern of each corresponds to their conserved function. In basal angiosperm, PI-like genes usually show broad expression zones in floral organs. For example, HoPI_1 and HoPI_3 from Hedyosmum orientale are expressed in all floral organs including the bracts and carpels. In addition, HoPI_1 mimics the endogenous Arabidopsis PI and fully rescues both petal and stamen development in Arabidopsis pi-1 mutants, while HoPI_3 only rescues petal development, and partially rescues stamen development [6]. In another basal angiosperm Magnolia wufengensis, the PI ortholog MawuPI was only able to rescue filament-like structures in the third whorl, but failed to rescue petal development in 
the second whorl [7]. Together, these data provide interesting evidence of a B-function evolutionary scenario among early-diverging clades of the angiosperm.

Monocots are one of the major clades of angiosperm, and the Orchidaceae family is one of the most species-rich in this clade; it includes species with highly diversified and specialized floral shapes. Previous studies have indicated that floral diversity in orchids results from changes in the regulatory regions of the MADS-box genes followed by gene duplication events [8], and that PI-like genes work together with different AP3/AGL6 homologs involved in specifying sepal/petal/lip development in Oncidium and Phalaenopsis orchids [9]. Here, we isolated a PI ortholog, CyfaPI, from a Chinese traditional orchid C. faberi Rolfe. The flower of C. faberi consists of three whorls of floral organs, with three sepals in whorl 1, two petals and a lip in whorl 2, and a gynostemium fused by the male and female tissues in whorl 3 (Figure 1). CyfaPI transcripts are detected in all floral organs, but are absent in the leaves, which showed a different expression pattern to PI-like genes from eudicots. Further functional analysis revealed that CyfaPI is able to fully complement petals in whorl 2, but can only rescues filament-like structures in whorl 3 of flowers in transformed Arabidopsis pi-1 mutants. Our data may provide the clue to help trace the gynostemium development program and evolution in orchids.

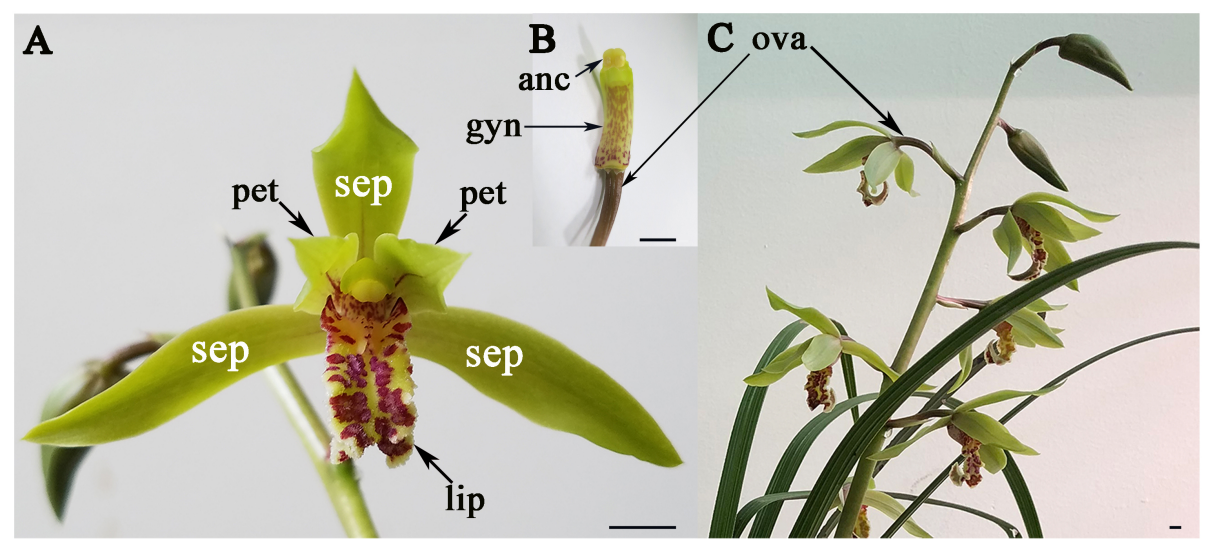

Figure 1. Flower and inflorescence of Cymbidium faberi. (A) Flower of C. faberi; (B) The gynostemium and ovary of $C$. faberi; (C) The inflorescence of $C$. faberi. Sepals (sep), petals (pet), lips (lip), anther cap (anc), gynostemium (gyn), ovary (ova). Bars: $5 \mathrm{~mm}$.

\section{Results}

\subsection{Cloning and Sequence Alignments of CyfaPI}

The CyfaPI (Cymbidium faberi PISTILLATA) cDNA is 932 bp with a 633 bp ORF (Open Reading Frame) encoding a 210 amino acid (aa) protein (Genbank accession number: MK450600.1). Putative protein alignment and a phylogenetic analysis grouped CyfaPI into the GLO/PI lineage (Figure 2). In addition, CyfaPI is nested within a clade of orthologs from other monocots and is most closely related to orthologs from the Orchidaceae, such as Oncidium OMADS8 (97.14\% identity) (Figure 2A). Computational translation shows that CyfaPI is a putative transcriptional regulator that contains a highly conserved 57 aa MADS-box domain (1-57) at the N-terminal region, a less conserved 64 aa K domain (87-150) in the middle region and a highly conserved 16 aa PI motif (193-208) in the variable $C$ terminal region (151-210). Moreover, CyfaPI contains three putative amphipathic $\alpha$-helices, K1(87-108), K2 (121-135) and K3 (143v167) subdomains that could potentially mediate protein interaction (Figure 2B) [10-12]. In addition, CyfaPI is 55.56\%, 55.66\%, 59.05\%, 60.85\%, 71.09\%, $64.29 \%, 74.53 \%, 79.05 \%$, and $67.14 \%$ identified to PI from A. thaliana, TrPI from T. rupestris, MtPI from $M$. truncatula, CabuPI from C. bungei, HoPI_1 and HoPI_3 from H. orientale, MAwuPI from M. wufengensis, and LMASDS8 and LMADS9 from Lilium longiflorum, respectively (Figure 2B). 
A

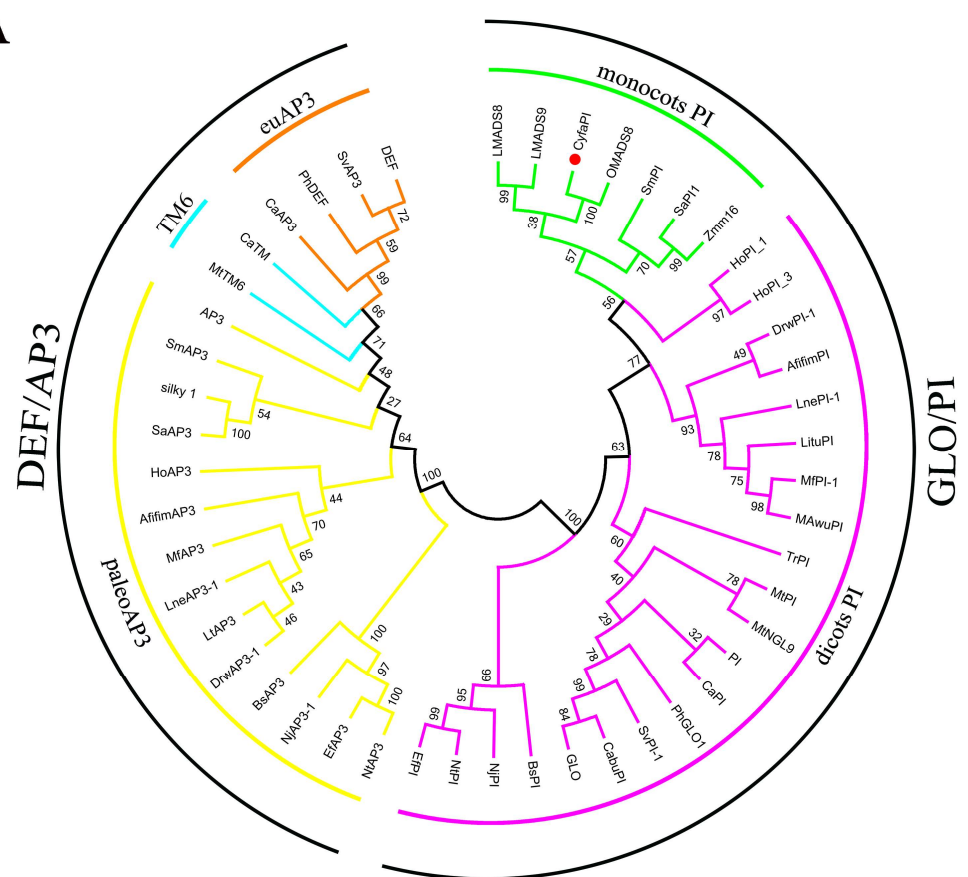

B
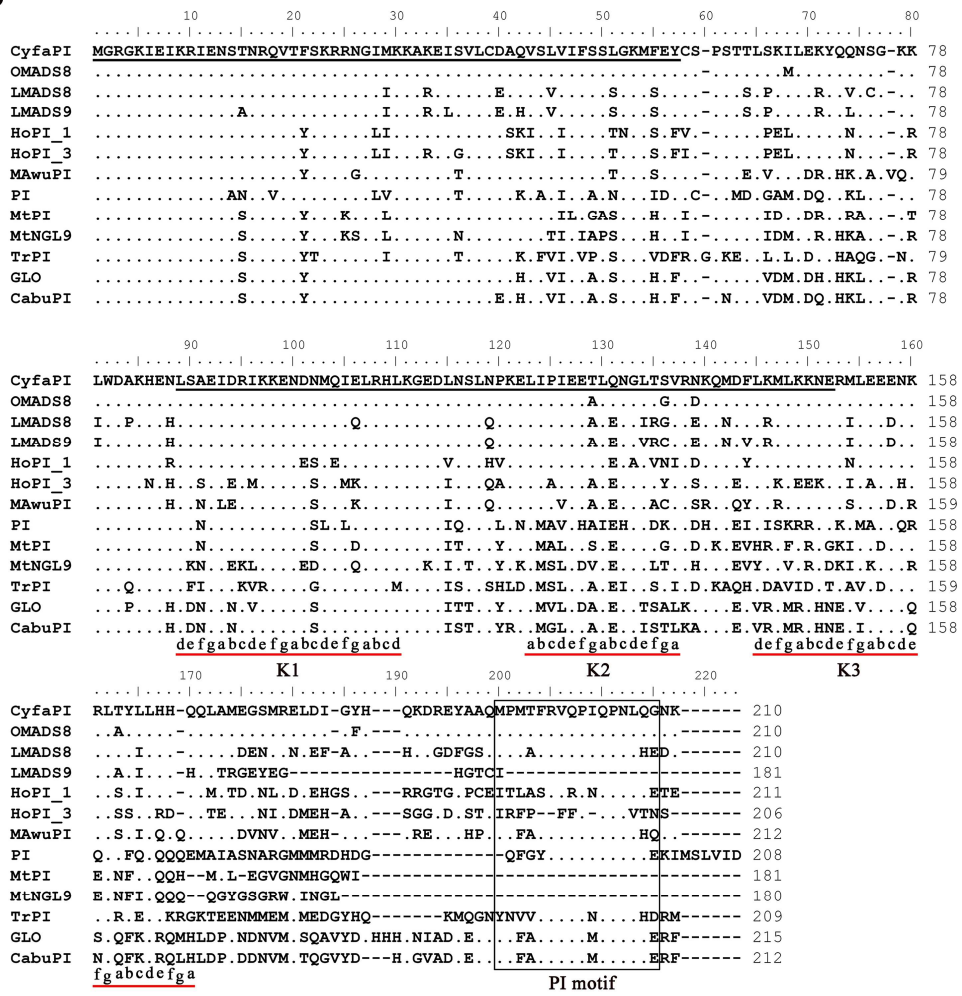

Figure 2. Phylogenetic analysis and sequence alignments of CyfaPI with B-class MADS-box proteins from other plant species. (A) Phylogenetic analysis of B-class MADS-box proteins; (B) Sequence alignments of CyfaPI with PI-like proteins from other plant species; the first underline indicates the MADS-box domain and the second the $\mathrm{K}$ domain. The PI motif in the C-terminal region is boxed. Dots represent amino acids (aa) identical to CyfaPI. Dashes indicate gaps inserted to improve the sequence. The K1, K2 and K3 helices with the (abcdefg)n heptad repeats are also underlined.

\subsection{Expression Analyses of CyfaPI in C. faberi}

CyfaPI expression was restricted to floral organs, such as sepals, petals, lips, anther caps, gynostemia and ovaries, and was absent in juvenile leaves (Figure 3). In addition, the highest CyfaPI 
expression was observed in gynostemia, where the expression was significantly higher than that in sepals, petals, lips, anther caps and ovaries $(L S D, p<0.05)$. Moreover, there was no significant differences in CyfaPI expression in the sepals and petals, where the CyfaPI expression in both these tissues was significantly higher than that in the lips, anther caps and ovaries $(L S D, p<0.05)$. The lowest CyfaPI expression was detected in anther caps, where the expression was considerably lower than that in sepals, petals, gynostemia and ovaries $(L S D, p<0.05)$, but showed no significant difference to CyfaPI expression in lips ( $L S D, p>0.05$ ) (Figure 3A). CyfaPI expression was also detectable in floral buds at $5 \mathrm{~mm}$ (early stages of flower differentiation), $8 \mathrm{~mm}$ (seasonal dormancy of floral buds), $10 \mathrm{~mm}$ (breaking dormancy and further development), $15 \mathrm{~mm}, 20 \mathrm{~mm}, 25 \mathrm{~mm}$ and at bud opening (Figure 3B). Moreover, CyfaPI expression became detectable during the early stages of flower differentiation and increased until the seasonal dormancy of floral buds. In addition, with the breaking of flower dormancy and further development, $\mathrm{CyfaPI}$ expression increased continuously and achieved its highest level $(L S D, p<0.05)$ in floral buds of $25 \mathrm{~mm}$ when it reached maturity, and then began to drop after the floral buds opened.

(A)

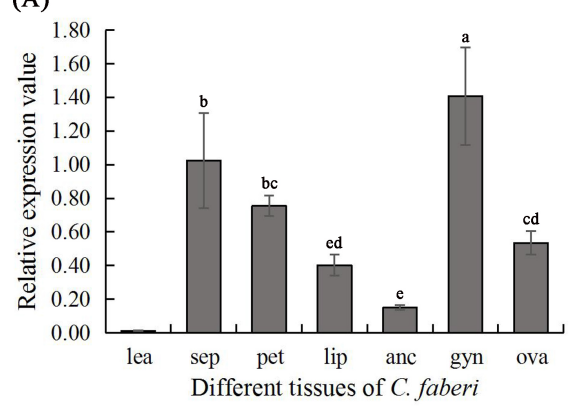

(B)

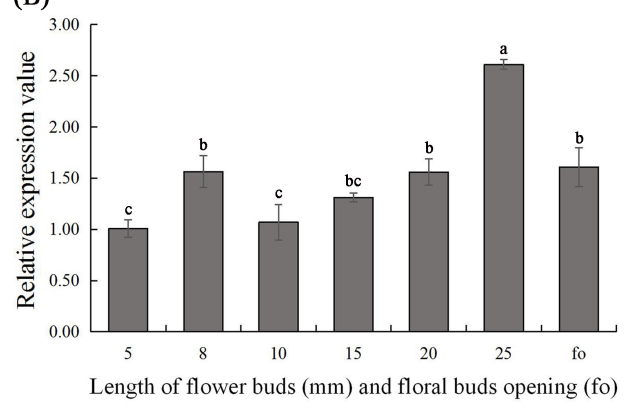

Figure 3. CyfaPI expression was detected by RT-qPCR in different tissues (A) and various stages of flower development (B) in C. faberi. (A) Expression of CyfaPI in the juvenile leaves (lea), sepals (sep), petals (pet), lips (lip), anther caps (anc), gynostemia (gyn) and ovaries (ova) with the C. faberi ACTIN gene used as the internal control; (B) Expression of CyfaPI at various stages of flower development. Different letters indicate statistically significant differences.

\subsection{Ectopic Expression of CyfaPI in Arabidopsis pi-1 Mutant}

To further determine the role of CyfaPI in flower development, we attempted to rescue the loss-of-function Arabidopsis pi mutant (pi-1) via the expression of a CyfaPI transgene. Binary vectors carrying 35S::CyfaPI were introduced into Arabidopsis by Agrobacterium-mediated transformation. The transgenic Arabidopsis lines were confirmed by RT-qPCR and the mutant allele was identified by dCAPS genotyping (Figure S1). Seven wild-type, 11 homozygous pi-1 and 14 heterozygote pi-1 independent transgenic Arabidopsis lines were obtained. Phenotypes of the transgenic lines were assayed in a homozygous pi-1 mutant background to evaluate whether the CyfaPI transgene could act as a substitute for the endogenous PI gene in regulating petal and stamen development. Phenotypes of transgenic lines were also analyzed in a wild-type Arabidopsis background to evaluate the function of CyfaPI in the floral development (Table 1). In addition, the CyfaPI expression in homozygous pi-1 mutants and wild type transgenic Arabidopsis with different phenotypes was also determined (Figure 4).

Among the 11 35S:: CyfaPI homozygous pi-1 transformants, 3 (27.27\%) lines showed strong complementation phenotypes with flowers consisting of four concentric whorls of floral organs. The flower has 4 petaloid sepals in whorl 1 (outmost ring), 4 petals in whorl 2, 3 (or 4) filament-like organs without anther attachment in whorl 3 and a normal gynoecium in whorl 4 (innermost central area) (Figure 5G,H). Two (18.18\%) lines displayed medium complementation phenotypes that produced flowers with four concentric whorls of floral organs. There were 4 normal sepals in whorl 1, 4 petals in whorl 2, 3 (or 4) filament-like organs without anther attachment in whorl 3 and a normal gynoecium in whorl 4 of flowers of this complementation group (Figure $5 \mathrm{~K}, \mathrm{~L}$ ). Six lines (54.55\%) failed to 
display a phenotype complementation and produced flowers similar to homozygous pi-1 mutant flowers. In addition, the flower phenotypes of transgenic Arabidopsis in heterozygous pi-1 mutant and the wild-type background were also assayed, and almost all the flower-phenotype changes in transgenic Arabidopsis in heterozygous PI/pi-1 background resembled the flower phenotypes displayed by wild-type lines expressing the introduced transgene. Among a total of $735 S::$ CyfaPI wild-type transgenic Arabidopsis, 3 (42.86\%) lines showed severe phenotype changes and produced flowers with sepals converted into petaloid organs in whorl 1 (Figure 5E,F), and $3(42.86 \%)$ lines displayed medium phenotype changes and ha flowers with the first whorl sepal partly converted into petal (Figure 5I,J). In addition, the CyfaPI expression levels corresponded to the flower-phenotype changes in transgenic plants (Figure 4). For example, CyfaPI expression levels in transgenic Arabidopsis lines in homozygous pi-1 background with strong complementation phenotypes $\left(\mathrm{T}_{1}-9 / p i-1, \mathrm{~T}_{1}-10 / p i-1\right)$ were significantly higher than that in homozygous $p i-1$ transgenic plants with medium complementation phenotypes $\left(\mathrm{T}_{1}-7 / p i-1, \mathrm{~T}_{1}-8 / p i-1\right)$ or without complementation of petal and stamen development $\left(\mathrm{T}_{1}-1 / p i-1, \mathrm{~T}_{1}-2 / p i-1\right)$ ( $L S D, p<0.05)$. Moreover, CyfaPI expression levels in homozygous pi-1 transgenic plants with medium complementation phenotypes $\left(\mathrm{T}_{1}-7 / p i-1, \mathrm{~T}_{1}-8 / p i-1\right)$ were also observed to be significantly higher than that in homozygous $p i-1$ transgenic plants without phenotype complementation $\left(\mathrm{T}_{1}-1 / p i-1, \mathrm{~T}_{1}-2 / p i-1\right)$ $(L S D, p<0.05)$. Similar results were also observed in wild-type transgenic Arabidopsis. Furthermore, three interesting flower phenotypes were also found in the 35S:: CyfaPI transgenic Arabidopsis in the heterozygous PI/pi-1 background. Specifically, one line produced significantly larger sized flowers, and another line produced flowers with five petals in whorl 2. The third line produced flowers with six petals in whorl 2 (Figure 6).
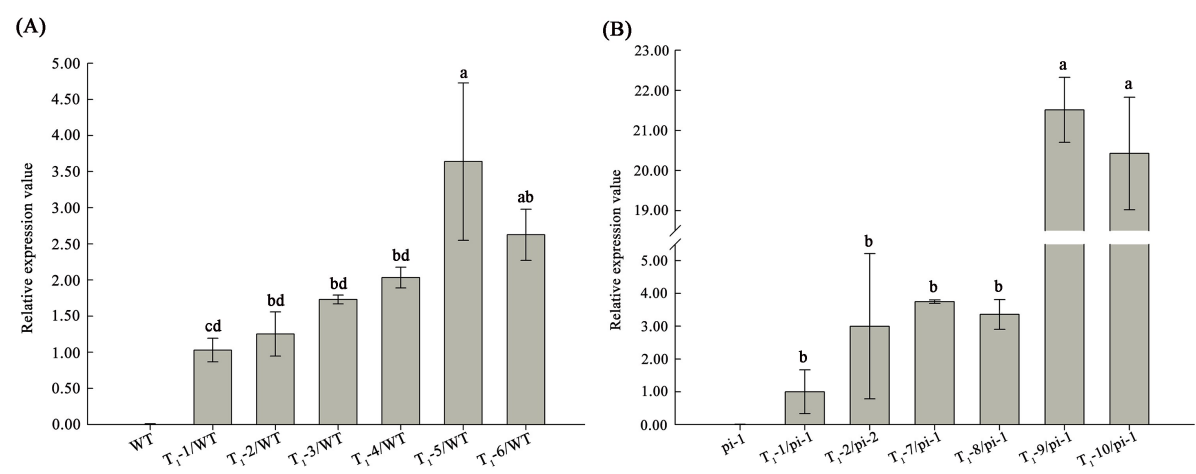

Figure 4. The CyfaPI expression was detected by RT-qPCR in the $\mathrm{T}_{1}$ transgenic plants in wild-type and homozygous Arabidopsis pi-1 mutant background. (A) CyfaPI expression was detected in wild-type transgenic Arabidopsis; (B) CyfaPI expression was detected in homozygous pi-1 mutant transgenic Arabidopsis. Different letters indicate statistically significant differences.

Wild-type (WT) Arabidopsis; $\left(\mathrm{T}_{1}-1 / \mathrm{WT}\right) 35 \mathrm{~S}::$ CyfaPI transgenic line without phenotypic changes; $\left(\mathrm{T}_{1}-2 / \mathrm{WT}, \mathrm{T}_{1}-3 / \mathrm{WT}, \mathrm{T}_{1}-4 / \mathrm{WT}\right) 35 \mathrm{~S}::$ CyfaPI transgenic lines displayed weak phenotype changes in floral structure; $\left(\mathrm{T}_{1}-5 / \mathrm{WT}, \mathrm{T}_{1}-6 / \mathrm{WT}\right) 35 \mathrm{~S}::$ CyfaPI transgenic lines displayed severe phenotype changes in floral structure; (pi-1) Homozygous pi-1 mutant Arabidopsis; $\left(\mathrm{T}_{1}-1 / p i-1, \mathrm{~T}_{1}-2 / p i-1\right) 35 S:: C y f a P I$ transgenic lines without phenotype complementation; $\left(\mathrm{T}_{1}-7 / p i-1, \mathrm{~T}_{1}-8 / p i-1\right) 35 S:: C y f a P I$ transgenic lines displayed medium complementation phenotypes; and ( $\left.\mathrm{T}_{1}-9 / p i-1, \mathrm{~T}_{1}-10 / p i-1\right) 35 \mathrm{~S}:: \mathrm{CyfaPI}$ transgenic lines showed strong complementation phenotypes. 

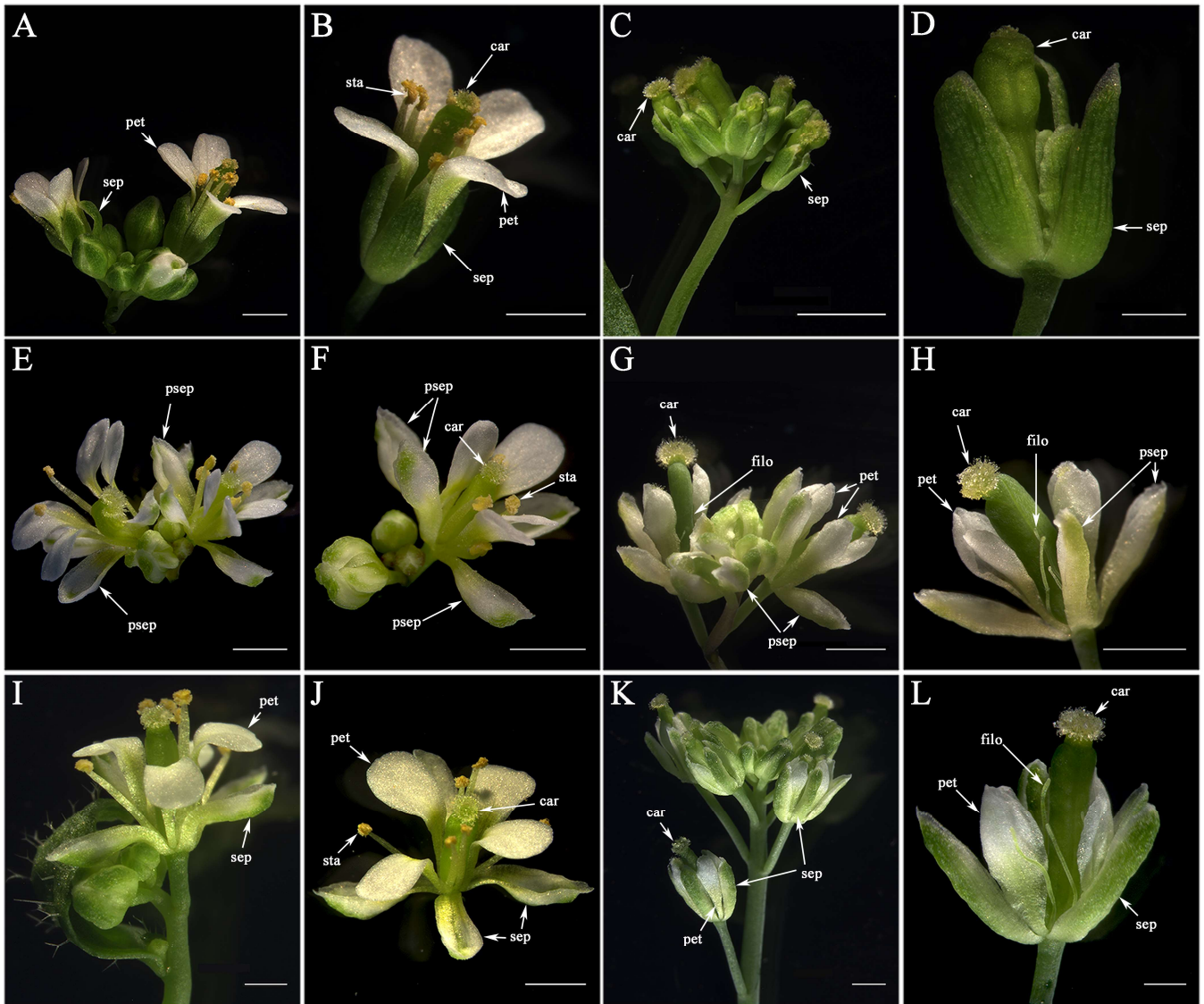

Figure 5. Phenotypes comparison of the wild-type, homozygous pi-1 mutant, 35S:: CyfaPI transgenic Arabidopsis under a wild-type and homozygous pi-1 mutant background. (A) Inflorescence of wild-type Arabidopsis; (B) Wild-type Arabidopsis flower with normal 4 concentric whorls of floral organs (4 sepals in whorl 1, 4 petals in whorl 2, 6 stamens in whorl 3 and a gynoecium in whorl 4); (C) Inflorescence of homozygous pi-1 mutant Arabidopsis; (D) Flower of homozygous pi-1 mutant Arabidopsis with two outer whorl sepals and one inner whorl gynoecium; (E) Inflorescence of 35S:: CyfaPI transgenic Arabidopsis in wild-type background displaying severe phenotype changes; (F) Flower of 35S:: CyfaPI transgenic Arabidopsis in wild-type background showing severe phenotype changes; $(\mathbf{G})$ Inflorescence of 35S:: CyfaPI homozygous pi-1 transgenic Arabidopsis showing strong complementation phenotypes; (H) Flower of 35S:: CyfaPI homozygous pi-1 transgenic Arabidopsis showing strong complementation phenotypes; (I) Inflorescence of 35S:: CyfaPI transgenic Arabidopsis in wild-type background displaying medium phenotype changes; (J) Flower of 35S:: CyfaPI transgenic Arabidopsis in wild-type background showing medium phenotype changes; (K) Inflorescence of 35S:: CyfaPI homozygous pi-1 transgenic Arabidopsis showing medium complementation phenotypes; (L) Flower of 35S:: CyfaPI homozygous pi-1 transgenic Arabidopsis showing medium complementation phenotypes. sepal (sep), petaloid sepal (psep), petals (pet), stamen (sta), filament-like organ (filo), carpel (car); Bars: (C) 2 mm; (A, B, E, F, G, H, J, K) $1 \mathrm{~mm}$; (D, I, L) $500 \mu \mathrm{m}$.

Table 1. Summary of different phenotypes of transgenic lines.

\begin{tabular}{ccc}
\hline Transgenic Lines & Phenotype of Flower & No. Plants \\
\hline \multirow{3}{*}{ 35S:: CyfaPI homozygous pi-1 transformants } & strong complementation & $3(27.27 \%)$ \\
& medium complementation & $2(18.18 \%)$ \\
& no complementation & $6(54.55 \%)$ \\
35 :: CyfaPI wild-type transformants & severe phenotype change & $3(42.86 \%)$ \\
& weak phenotype change & $3(42.86 \%)$ \\
& no phenotype change & $1(14.28 \%)$ \\
\hline
\end{tabular}




\section{Discussion}

Molecular studies in model species have indicated that the function of floral MADS-box genes is highly correlated with their expression patterns; therefore, the expression patterns of MADS-box genes in other species may be a good predictor [13]. In Arabidopsis, PI works together with another B-function protein APETALA3 (AP3) to specify petal and stamen identity. Therefore, high levels of PI expression are restricted to petals and stamens [14]. Moreover, PI orthologs, such as MtPI from M. truncatula [3,15], TrPI from T. rupestris [4], and CabuPI from C. bungei [5], show conserved expression patterns and functions in these core eudicots. However, some PI orthologs from basal angiosperm species, such as $H$. orientale and $M$. wufengensis, have shown broad expression zones in floral organs and have been demonstrated to have different functions [6,7]. H. orientale, HoPI_1 and HoPI_3 showed similar expression zones, but different expression levels. In addition, HoPI_1 fully rescued both petal and stamen development in Arabidopsis pi-1 mutants, while HoPI_3 could only rescue petal development as well as partially rescuing stamen development [6]. However, the MawuPI from M. wufengensis only partly rescued the stamen development in Arabidopsis pi-1 mutants [7]. In addition, some monocots' PI orthologs were also found to have expressions that extended outwards from the first whorl perianth [16,17]. For examples, two L. longiflorum PI orthologs, LMASDS8 and LMADS9, are expressed persistently in all the perianth whorls during flower development, but only in the stamens of young flower buds. Moreover, both genes could fully rescue petal development, but failed to complement stamen development in the Arabidopsis pi-1 mutant [16]. Together, these data suggest that changes in expression domains or an altered sequence within the $P I$ orthologs may contribute to functional divergence. O. Gower Ramsey OMADS8, an orchid PI ortholog, was expressed in all floral organs and vegetative leaves, and was able to fully complement petal and stamen development in the Arabidopsis pi-1 mutant [17,18]. Moreover, the Oncidium PI ortholog has been demonstrated to work together with AP3-like and AGL6-like proteins to regulate sepal/petal/lip development and differentiation [9]. Similar expression and function of $P I$ ortholog was also observed in Phalaenopsis [9,19-21]. Some PI orthologs from other Orchidaceae species, such as Liparis distans, Phaius tankervilliae, Dendrobium Spring were also found to be expressed in all floral whorls [22]. Interestingly, two copied orchid PI orthologs are present in Orchis italica, and both genes are expressed in all floral organs. In addition, the highest expression level of both genes is detected in the lateral inner tepals of the immature inflorescence, and is detected in the lips of the mature inflorescence; while the continued lowest expression level of both genes is found in the columns from immature to mature inflorescence [23,24]. In our study, C. faberi CyfaPI expression was found in all floral organs, but was absent in leaves. In addition, the highest expression level of CyfaPI was present in the gynostemia, and the lowest expression level was in the anther caps. A relatively high expression level of CyfaPI was also found in the sepals and petals (Figure 3A). That is, CyfaPI showed a different expression pattern with the PI orthologs from closely related species, such as L. longiflorum and O. Gower Ramsey. Moreover, protein sequence alignments suggested that CyfaPI is 79.05\%, 67.14\%, $97.14 \%$ identified with LMASDS8, LMADS9 from L. longiflorum, and OMADS8 from O. Gower Ramsey, respectively (Figure 2B). Furthermore, ectopic expression of CyfaPI in Arabidopsis pi-1 mutant fully rescued petal development, and complemented filament-like structure but failed to complement anther development in the stamen whorl. Together, these data suggest that changes in expression zones or an altered sequence within the PI orthologs in different groups of monocots may contribute to floral morphology changes. In $C$. faberi, high CyfaPI expression was found in the perianth (sepal and petal) and gynostemium (fused organ of filament and column). In addition, complementary loss-of-function alleles showed that $C y f a P I$ could rescue petal and filament development in Arabidopsis pi-1 mutant. These results suggest that CyfaPI is mainly responsible for perianth and gynostemium development in C. faberi.

Specifically, one line of transgenic Arabidopsis in heterozygous PI/pi-1 background produced noticeably larger sized flower, and another line produced flowers with five petals in whorl 2 . The third line produced flowers with six petals in whorl 2 (Figure 6). Perhaps the future challenge is to uncover how C. faberi PI ortholog, CyfaPI, is involved in increasing petal number and the size of floral organs. 
In addition, our experiment suggests that $C y f a P I$ holds the potential for biotechnical engineering to develop new ornamental plant varieties of orchids with interesting and valuable traits.
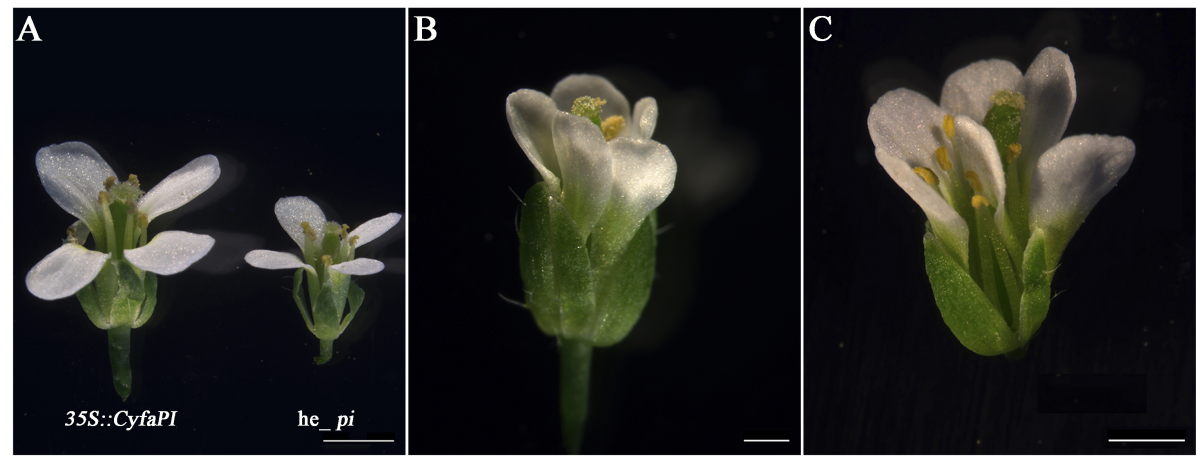

Figure 6. Interesting flower phenotypes of 35S::CyfaPI transgenic PI/pi-1 Arabidopsis. (A) The large flower of 35S::CyfaPI transgenic PI/pi-1 Arabidopsis and the PI/pi-1 Arabidopsis flower (he_pi) with four sepals in whorl 1, four petals in whorls 2, six stamens in whorl 3 and a gynoecium in whorl 4; (B) 35S::CyfaPI transgenic PI/pi-1 Arabidopsis producing flower with five petals in whorl 2; (C) 35S::CyfaPI transgenic PI/pi-1 Arabidopsis producing flower with six petals in whorl 2. Bars: (A) $5 \mathrm{~mm}$; (C) $1 \mathrm{~mm}$; (B) $500 \mu \mathrm{m}$.

\section{Materials and Methods}

\subsection{Plant Material}

Cymbidium faberi plants were grown in ceramic flowerpots $(\varnothing 25.5 \times 26 \mathrm{~cm})$ full with orchid substrates in the Botanical Garden of Yangtze University under natural conditions in Jingzhou city, Hubei Province. Juvenile leaves, sepals, petals, lips, anther caps, gynostemia and ovaries were sampled when the flower buds opened, immediately frozen in liquid nitrogen and stored at $-80{ }^{\circ} \mathrm{C}$ until use. In addition, floral buds measuring $5 \mathrm{~mm}$ (early stages of flower differentiation), $8 \mathrm{~mm}$ (seasonal dormancy of floral buds), $10 \mathrm{~mm}$ (breaking flower dormancy and further development), $15 \mathrm{~mm}, 20 \mathrm{~mm}, 25 \mathrm{~mm}$ long and opening floral buds were also sampled. The pi-1 mutant Arabidopsis (CS77) seeds were obtained from the ABRC (Arabidopsis Biological Resource Center, ABRC) at the Ohio State University, USA.

\subsection{Isolation and Characterization of PI Ortholog from C. faberi}

Total RNA was extracted from different tissues as described above by using an EASYspin Plant RNA Kit (Aidlab, Beijing, China) according to the manufacturer's protocols. First-strand cDNA was synthesized from $1 \mu \mathrm{g}$ of DNase I-treated total RNA with an oligo (dT)18 adaptor primer and M-MLV Reverse Transcriptase (TaKaRa, Shiga, Japan). The CyfaPI 3' end cDNA sequences were amplified with gene-specific forward primer GSPPI (5'-ATCAATAGGCAGGTGACCTTCTC-3') and $3^{\prime}$ RACE Outer Primer (5'-TACCGTCGTTCCACTAGTGATTT- $3^{\prime}$ ) following the manufacturer's protocols as described in the $3^{\prime}$-full RACE Core Set Ver. 2.0 kit (TaKaRa, Shiga, Japan), but with $45 \mathrm{~s}$ annealing at $57{ }^{\circ} \mathrm{C}$. The full length of CyfaPI cDNA was amplified with the forward primer CyfaPIF (5'- CTTTTTGCTTCTGTTCTTGAGATC - $\left.3^{\prime}\right)$ and the reverse primer CyfaPIR (5'GCACGCACATATGTCATAATCATC-3'), and the PCR amplification procedure followed Liu et al. [25]. The primer design was based on the sequence of PI/GLO orthologs, CyenPI (Genbank accession numbers: JQ326259.1) from the closely related species C. ensifolium (Orchidaceae). Putative protein sequences of CyfaPI and 85 B-class MADS-box proteins in NCBI Genbank (Table S1) from other angiosperms species were selected for constructing phylogenetic trees. The B-class MADS-box proteins were identified based on previously published papers and a BLAST search in the Genbank sequence database. Phylogenetic trees were constructed with MEGA5.0 software using the neighbor-joining method described by Tamura et al. [26]. with the default setting, and bootstrap values were based on 
1000 replicates. The full-length CyfaPI proteins consisting of complete MADS, I, K and C domains were aligned with BioEdit 7.0.9 software using the ClustalW program with default settings.

\subsection{Expression Analysis of CyfaPI}

For RT-qPCR analysis, total RNAs from juvenile leaves, sepals, petals, lips, anther caps, gynostemia, ovaries, floral buds of different length $(5 \mathrm{~mm}, 8 \mathrm{~mm}, 10 \mathrm{~mm}, 15 \mathrm{~mm}, 20 \mathrm{~mm}, 25 \mathrm{~mm})$ and opening floral buds were extracted using the procedure described above. DNA free total RNA and first-strand cDNA were prepared by using the HiScript ${ }^{\circledR}$ II Q RT SuperMix for qPCR kit (Vazyme, Nanjing China) following the manufacturer's protocols. The RT-qPCR of three biological replicates was performed with the gene-specific forward primer qCyfaPIF(5' - CACATACCTATTGCACCATC-3') and the gene-specific reverse primer qCyfaPIR(5' - GGTCATTGGCATCTGAGCTG- $\left.3^{\prime}\right)$ on the Line-Gene 9600 Plus Real-time PCR Detection System, with SYBR green I for transcript measurements. Amplification of C. faberi actin (Genbank accession numbers: JN177719.1) fragment with specific primers qCyfaactinF (5'- AAGACTTACACCAAGCCGAAGAAGATC- $\left.3^{\prime}\right)$ and qCyfaactinR (5'- CCAGCTCCACAGGTTGCGTTAG - $3^{\prime}$ ) was used as the internal control. The PCR procedure was as follows: $95^{\circ} \mathrm{C}$ for $30 \mathrm{~s}$, followed by 40 cycles of $95^{\circ} \mathrm{C}$ for $10 \mathrm{~s}, 60^{\circ} \mathrm{C}$ for $30 \mathrm{~s}$, which was followed by melt curve stages of $95^{\circ} \mathrm{C}$ for $15 \mathrm{~s}, 60^{\circ} \mathrm{C}$ for $60 \mathrm{~s}$, and $95^{\circ} \mathrm{C}$ for $15 \mathrm{~s}$. The experiments were repeated three times for each sample. Relative expression levels were calculated based on the $2^{-\Delta \Delta \mathrm{Ct}}$ method [27].

\subsection{Ectopic Expression of CyfaPI in Arabidopsis pi-1 Mutant and Function Analysis}

CyfaPI cDNAs containing full-ORF (open reading frame) were cloned into the pBI121 vector with Xba I and Xma I restriction enzymes in the sense orientation. The 35S:: CyfaPI constructs were then transformed into heterozygous PI/pi-1 Arabidopsis with the floral-dip method suggested by Clough and Bent [28]. The $\mathrm{T}_{1}$ seeds were selected and seedlings were cultivated by referring to the methods described by Li et al. [29]. Moreover, the positive transgenic lines were confirmed by RT-qPCR as described above with the same CyfaPI-gene-specific primers qCyfaPIF and qCyfaPIR suggested above. For RT-qPCR, the $A$. thaliana $\beta$-actin (Genbank accession numbers: NM_116090.3) fragment was amplified as the internal control with the forward primer qactinF ( $5^{\prime}$ - AAGACTTACACCAAGCCGAAGAAGATC $-3^{\prime}$ ) and the reverse primer qactinR ( $5^{\prime}$ - CCAGCTCCACAGGTTGCGTTAG -3'). Homozygous pi-1 transformants were identified by dCAPS genotyping following the method suggested by Lamb and Irish [30]. Phenotypes of transgenic Arabidopsis in homozygous pi-1 mutant background and wild-type background were assayed after flowering, respectively.

\subsection{Statistical Analyses}

All experiments were carried out with three biological replicates, and data were expressed as mean $\pm \mathrm{SE}$ (standard error). Statistical significance was determined using one-way ANOVA with the LSD (Least significant difference) test at $p<0.05$ level in SPSS 19.0. $P$ values of less than 0.05 were regarded as statistically significant.

Supplementary Materials: Supplementary materials can be found at http://www.mdpi.com/2073-4395/9/8/425/s1. Figure S1: Genotyping and phenotype of wild-type, homozygous pi-1 mutant and heterozygous PI/pi-1 Arabidopsis. Supplementary Table S1: Information on sequences selected for alignments and phylogenetic analyses from NCBI GenBank.

Author Contributions: Z.-X.L. designed the experiments, provided project supervision and revised the manuscript, Y.F. conducted the experiments and wrote the draft manuscript. All authors approved the final draft of the manuscript.

Funding: This research was supported by the Excellent Master Dissertation Cultivation Program of Yangtze University (YS2018049).

Conflicts of Interest: The authors declare no conflict of interest. 


\section{Abbreviations}

$\begin{array}{ll}\text { BLAST } & \text { Basic local alignment search tool } \\ \text { PCR } & \text { Polymerase chain reaction } \\ \text { RT-qPCR } & \text { Quantitative reverse transcription PCR } \\ \text { LSD } & \text { Least significant difference }\end{array}$

\section{References}

1. Heijmans, K.; Morel, P.; Vandenbussche, M. MADS-box Genes and Floral Development: The Dark Side. J. Exp. Bot. 2012, 63, 5397-5404. [CrossRef] [PubMed]

2. Monniaux, M.; Vandenbussche, M. How to Evolve a Perianth: A Review of Cadastral Mechanisms for Perianth Identity. Front. Plant Sci. 2018, 9, 1573. [CrossRef] [PubMed]

3. Hecht, V. Duplicate MADS genes with split roles. J. Exp. Bot. 2016, 67, 1609-1611. [CrossRef] [PubMed]

4. Lü, S.; Fan, Y.; Liu, L.; Liu, S.; Zhang, W.; Meng, Z. Ectopic expression of TrPI, a Taihangia rupestris (Rosaceae) PI ortholog, causes modifications of vegetative architecture in Arabidopsis. J. Plant Physiol. 2010, 167, 1613-1621. [CrossRef] [PubMed]

5. Jing, D.; Xia, Y.; Chen, F.; Wang, Z.; Zhang, S.; Wang, J. Ectopic expression of a Catalpa bungei (Bignoniaceae) PISTILLATA homologue rescues the petal and stamen identities in Arabidopsis pi-1 mutant. Plant Sci. 2015, 231, 40-51. [CrossRef] [PubMed]

6. Liu, S.; Sun, Y.; Du, X.; Xu, Q.; Wu, F.; Meng, Z. Analysis of the APETALA3- and PISTILLATA-like genes in Hedyosmum orientale (Chloranthaceae) provides insight into the evolution of the floral homeotic B-function in angiosperms. Ann. Bot. 2013, 112, 1239-1251. [CrossRef] [PubMed]

7. Liu, W.; Shen, X.; Liang, H.; Wang, Y.; He, Z.; Zhang, D.; Chen, F. Isolation and Functional Analysis of PISTILLATA Homolog from Magnolia wufengensis. Front. Plant Sci. 2018, 9, 1743. [CrossRef]

8. Aceto, S.; Gaudio, L. The MADS and the Beauty: Genes Involved in the Development of Orchid Flowers. Curr. Genom. 2011, 12, 342-356. [CrossRef]

9. Hsu, H.F.; Hsu, W.H.; Lee, Y.I.; Mao, W.T.; Yang, J.Y.; Li, J.Y.; Yang, C.H. Model for perianth formation in orchids. Nat. Plants 2015, 1, 15046. [CrossRef]

10. Riechmann, J.L.; Krizek, B.A.; Meyerowitz, E.M. Dimerization specificity of Arabidopsis MADS domain homeotic proteins APETALA1, APETALA3, PISTILLATA, and AGAMOUS. Proc. Natl. Acad. Sci. USA 1996, 93, 4793-4798. [CrossRef]

11. Yang, Y.; Jack, T. Defining subdomains of the K domain important for protein-protein interactions of plant MADS proteins. Plant Mol. Biol. 2004, 55, 45-59. [CrossRef] [PubMed]

12. Kim, S.; Yoo, M.J.; Albert, V.A.; Farris, J.S.; Soltis, P.S.; Soltis, D.E. Phylogeny and diversification of B-function MADS-box genes in angiosperms: Evolutionary and functional implications of a 260-million-year-old duplication. Am. J. Bot. 2004, 91, 2102-2118. [CrossRef] [PubMed]

13. Ma, H.; Depamphilis, C. The ABCs of Floral Evolution. Cell 2000, 101, 5-8. [CrossRef]

14. Goto, K.; Meyerowitz, E.M. Function and regulation of the Arabidopsis floral homeotic gene PISTILLATA. Genes Dev. 1994, 8, 1548-1560. [CrossRef] [PubMed]

15. Roque, E.; Fares, M.A.; Yenush, L.; Rochina, M.C.; Wen, J.; Mysore, K.S.; Gomez-Mena, C.; Beltrán, J.P.; Cañas, L.A. Evolution by gene duplication of Medicago truncatula PISTILLATA-like transcription factors. J. Exp. Bot. 2016, 67, 1805-1817. [CrossRef] [PubMed]

16. Chen, M.K.; Hsieh, W.P.; Yang, C.H. Functional analysis reveals the possible role of the C-terminal sequences and PI motif in the function of lily (Lilium longiflorum) PISTILLATA (PI) orthologues. J. Exp. Bot. 2012, 63, 941-961. [CrossRef] [PubMed]

17. Chang, Y.Y.; Kao, N.H.; Li, J.Y.; Hsu, W.H.; Liang, Y.L.; Wu, J.W.; Yang, C.H. Characterization of the possible roles for B class MADS box genes in regulation of perianth formation in orchid. Plant Physiol. 2010, 152, 837-853. [CrossRef]

18. Mao, W.T.; Hsu, H.F.; Hsu, W.H.; Li, J.Y.; Lee, Y.I.; Yang, C.H. The C-Terminal Sequence and PI motif of the Orchid (Oncidium Gower Ramsey) PISTILLATA (PI) Ortholog Determine its Ability to Bind AP3 Orthologs and Enter the Nucleus to Regulate Downstream Genes Controlling Petal and Stamen Formation. Plant Cell Physiol. 2015, 56, pcv129. 
19. Tsai, W.C.; Lee, P.F.; Chen, H.I.; Hsiao, Y.Y.; Wei, W.J.; Pan, Z.J.; Chuang, M.H.; Kuoh, C.S.; Chen, W.H.; Chen, H.H. PeMADS6, a GLOBOSA/PISTILLATA-like Gene in Phalaenopsis equestris Involved in Petaloid Formation, and Correlated with Flower Longevity and Ovary Development. Plant Cell Physiol. 2005, 46, 1125-1139. [CrossRef]

20. Hsieh, M.H.; Lu, H.C.; Pan, Z.J.; Yeh, H.H.; Wang, S.S.; Chen, W.H.; Chen, H.H. Optimizing virus-induced gene silencing efficiency with Cymbidium mosaic virus in Phalaenopsis flower. Plant Sci. 2013, 201, $25-41$. [CrossRef]

21. Tsai, W.C.; Pan, Z.J.; Hsiao, Y.Y.; Jeng, M.F.; Wu, T.F.; Chen, W.H.; Chen, H.H. Interactions of B-class complex proteins involved in tepal development in Phalaenopsis orchid. Plant Cell Physiol. 2008, 49, 814-824. [CrossRef] [PubMed]

22. Pan, Z.J.; Cheng, C.C.; Tsai, W.C.; Chung, M.C.; Chen, W.H.; Hu, J.M.; Chen, H.H. The Duplicated B-class MADS-Box Genes Display Dualistic Characters in Orchid Floral Organ Identity and Growth. Plant Cell Physiol. 2011, 52, 1515-1531. [CrossRef] [PubMed]

23. Salemme, M.; Sica, M.; Gaudio, L.; Aceto, S. Expression pattern of two paralogs of the PI/GLO-like locus during Orchis italica (Orchidaceae, Orchidinae) flower development. Dev. Genes Evol. 2011, 221, 241-246. [CrossRef] [PubMed]

24. Cantone, C.; Gaudio, L.; Aceto, S. The PI/GLO-like locus in orchids: Duplication and purifying selection at synonymous sites within Orchidinae (Orchidaceae). Gene 2011, 481, 48-55. [CrossRef] [PubMed]

25. Liu, Z.; Zhang, D.; Liu, D.; Li, F.; Lu, H. Exon skipping of AGAMOUS homolog PrseAG in developing double flowers of Prunus lannesiana (Rosaceae). Plant Cell Rep. 2013, 32, 227-237. [CrossRef]

26. Tamura, K.; Peterson, D.; Peterson, N.; Stecher, G.; Nei, M.; Kumar, S. MEGA5: Molecular Evolutionary Genetics Analysis Using Maximum Likelihood, Evolutionary Distance, and Maximum Parsimony Methods. Mol. Biol. Evol. 2011, 28, 2731-2739. [CrossRef] [PubMed]

27. Livak, K.J.; Schmittgen, T.D. Analysis of relative gene expression data using real-time quantitative PCR and the 2(-Delta Delta C(T)) Method. Methods 2001, 25, 402-408. [CrossRef]

28. Clough, S.J.; Bent, A.F. Floral dip: A simplified method forAgrobacterium-mediated transformation ofArabidopsis thaliana. Plant J. 1998, 16, 735-743. [CrossRef]

29. Li, L.Y.; Fang, Z.W.; Liu, Z.X. Isolation and characterization of the C-class MADS-box gene from the distylous pseudo-cereal Fagopyrum esculentum. J. Plant Biol. 2017, 60, 189-198. [CrossRef]

30. Lamb, R.S.; Irish, V.F. Functional divergence within the APETALA3/PISTILLATA floral homeotic gene lineages. Proc. Natl. Acad. Sci. USA 2003, 100, 6558-6563. [CrossRef] 